\title{
Variación en la diversidad malacológica del mesolitoral rocoso en Playa Troncones, La Unión, Guerrero, México
}

\author{
Variation in the diversity of mollusks from the rocky intertidial of Playa Troncones, La Unión, \\ Guerrero, México
}

\author{
Pedro Flores-Rodríguez ${ }^{1 *}$, Rafael Flores-Garza ${ }^{1}$, Sergio García-Ibáñez y Arcadio Valdés-González² \\ ${ }^{1}$ Unidad Académica de Ecología Marina, Universidad Autónoma de Guerrero. Gran Vía Tropical 20, Frac. Las Playas, 39390 Acapulco, Guerrero. \\ ${ }^{2}$ Laboratorio de Acuacultura, Facultad de Ciencias Biológicas, Unidad B, Universidad Autónoma de Nuevo León. Cd. Universitaria, San Nicolás de \\ los Garza, Nuevo León. \\ *Correspondencia: acua_uag@yahoo.com
}

\begin{abstract}
Resumen. Se presenta un inventario de especies, frecuencia de aparición, dominancia simple, diversidad e importancia relativa acumulada de la malacofauna presente en el mesolitoral rocoso de Playa Troncones, Guerrero. Además, se analizan los cambios de la densidad y diversidad en relación con la precipitación pluvial en un ciclo anual. Se llevaron a cabo 5 muestreos trimestrales entre diciembre 2000 y diciembre 2001, utilizando transectos de $30 \mathrm{~m}$ de largo con $2 \mathrm{~m}$ de amplitud y cuadrantes de $1 \mathrm{~m}$ por lado. El área de muestreo fue de $20 \mathrm{~m}^{2}$. La riqueza fue similar a lo registrado en otro sitio del estado de Guerrero. Se determinaron como especies representativas de la comunidad a Brachidontes semilaevis, Nerita funiculata, Isognomon janus, Plicopurpura pansa, Nerita scabricosta, Petaloconchus complicatus y Nodilittorina aspera, siendo B. semilaevis y P. pansa las de mayor importancia por su abundancia y frecuencia de aparición. Los valores de diversidad corresponden a valores comúnmente encontrados en regiones tropicales. Se observó que al aumentar la precipitación total, la densidad promedio de los organismos tiende a disminuir al mismo tiempo que se incrementa la diversidad biológica.
\end{abstract}

Palabras clave: moluscos, costa rocosa, riqueza, diversidad, densidad, dominancia.

\begin{abstract}
This work introduces a species inventory giving the dominant species their frequency of occurrence, their diversity as well as the species' accumulated relative importance of the mollusks at Playa Troncones in Guerrero State, México. It also analyzes changes in mollusk density and diversity in comparison to the annual rainfall cycle. Between December 2000 and December 2001, five three-monthly samples were carried out using $20 \times 1 \mathrm{~m}^{2}$ quadrats taken randomly within a strip measuring $30 \times 2 \mathrm{~m}$ to give a total sampled area of $20 \mathrm{~m}^{2}$. The richness found there was similar to that reported at another site in the State of Guerrero. The representative species of this community were Brachidontes semilaevis, Nerita funiculata, Isognomon janus, Plicopurpura pansa, Nerita scabricosta, Petaloconchus complicatus and Nodilittorina aspera. Brachidontes semilaevis and P. pansa were of greatest importance due to their abundance and frequency of appearance. The diversity index values recorded in the study area correspond to those found in tropical regions. In this study area, it was observed that an increase in rainfall produced a decrease in the average density of the organisms, whereas such increased precipitation produced an increase in the value of the biological diversity index.
\end{abstract}

Key words: mollusks, rocky shore, richness, diversity, density, dominant species.

\section{Introducción}

La costa rocosa es una zona expuesta a constantes inmersiones y emersiones, por lo que las condiciones para el desarrollo de la vida son bastante difíciles. Entre los

Recibido: 13 noviembre 2004; aceptado: 28 marzo 2007 organismos de esta zona suelen estar bien representados los moluscos, los cuales históricamente han estado asociados al hombre y a su desarrollo cultural.

En el estado de Guerrero, México, el conocimiento de los recursos faunísticos marinos es escaso, a pesar de que cuenta con una amplia zona costera consistente en $470 \mathrm{~km}$ de longitud (Carranza-Edwards et al., 1975), que abarca 
3 regiones marinas prioritarias para la conservación de la biodiversidad costera y oceánica de México, en las que la Comisión Nacional para el Conocimiento y Uso de la Biodiversidad (CONABIO) señala la falta de conocimiento en estas áreas (Arriaga et al., 1998).

La mayoría de los trabajos publicados sobre los moluscos del litoral del Pacífico mexicano son de carácter faunístico o taxonómico; algunos tratan aspectos de diversidad y variación a través del tiempo (Stuardo y Villarroel, 1976; Baqueiro y Stuardo, 1977; Baqueiro, 1979; Reguero y García, 1989; Holguín y González, 1989, 1994; Sevilla, 1995; Pérez y Ríos, 1998; Landa y Arciniega, 1998; Olavaria, 1999; Ríos et al., 2001) y otros han investigado acerca de la distribución y abundancia (Román et al., 1991; Esqueda et al., 2000; Villarroel et al., 2000).

Para el caso del intermareal rocoso de Guerrero se conocen estudios de riqueza y zonación de moluscos en las localidades de Acapulco (Villalpando, 1986; Delgado, 1989; García, 1994; Flores et al., 2003) y Zihuatanejo (Salcedo et al., 1988); no obstante, el conocimiento de los moluscos de esta región es aún insuficiente.

Esta investigación tiene como objetivos determinar la riqueza, frecuencia de aparición, densidad, dominancia simple y diversidad de las especies de la malacofauna, así como definir las de mayor importancia relativa acumulada y conocer los cambios en un ciclo anual. Además, analizar la variación de la densidad y diversidad en relación con la precipitación total mensual, en la zona mesolitoral rocosa de Playa Troncones en el periodo.

\section{Materiales y métodos}

Playa Troncones se ubica en la región de la Costa Grande municipio de la Unión, Guerrero (17 ${ }^{\circ} 47^{\prime} 35.0^{\prime \prime} \mathrm{N}$ y $101^{\circ} 44^{\prime} 46.6$ " O). La descripción de la playa se realizó de acuerdo con la carta geológica del Instituto Nacional de Estadística, Geografía e Informática (Zihuatanejo E14-7-10, escala 1:250,000), Mottana et al. (1980) y observaciones de campo registradas durante el transcurso de la investigación. Esta playa es de oleaje suave o protegida, compuesta por rocas sedimentarias y volcanosedimentarias del cuaternario, tipo aluvial y/o litoral, de tonalidades grises oscuras. Presenta montículos fijos de estructura compacta con gran cantidad de fisuras y grietas que durante la marea alta son parcialmente cubiertos. Según Briggs (1974), la zona de estudio pertenece a la Provincia Mexicana y de acuerdo con el sistema de clasificación climática de Köpen modificado por García (1981), se define como cálida-subhúmeda, con lluvias en verano (Aw, wi) y con 5 a $10 \%$ de lluvia invernal.
Lainformación sobre la precipitación pluvial mensual total durante el periodo en el que se desarrollaron las recolectas, fue proporcionada por la subestación climatológica La Unión de la Gerencia Regional Pacífico-Centro, de la Comisión Nacional del Agua.

Playa Troncones fue seleccionada para su estudio porque está incluida en un estudio sobre la diversidad malacológica de la costa rocosa del estado de Guerrero, que llevó a cabo la Unidad Académica de Ecología Marina de la Universidad Autónoma de Guerrero, México; además, es de fácil acceso y el impacto antropogénico que presenta, producto de la actividad turística y de los pobladores, es bajo.

Se realizaron 5 muestreos en el mesolitoral (Stephenson y Stephenson, 1949), uno cada trimestre, de diciembre de 2000 a diciembre de 2001, en una área de muestreo de 20 $\mathrm{m}^{2}$, definida por el método de especies-área (Brower et al., 1998). Se empleó un transecto de $30 \mathrm{~m}$ de largo por $2 \mathrm{~m}$ de ancho, paralelo a la línea de costa; la unidad de muestreo fue un cuadrante de $1 \mathrm{~m}$ por lado. El muestreo fue sistemático (Scheaffer et al., 1987) y el punto de inicio se seleccionó al azar.

Se recolectaron los moluscos que se encontraron dentro de los cuadrantes y se cuantificaron e identificaron. La ubicación taxonómica se confirmó en el laboratorio mediante el uso de claves especializadas y el esteroscopio con base en el análisis conquiliológico de Keen (1971), y la nomenclatura se actualizó de acuerdo con Skoglund (1991, 1992). Los ejemplares recolectados se depositaron en la colección de moluscos del Laboratorio de Investigación en Ecología Costera y Sustentabilidad, Unidad Académica de Ecología Marina, Universidad Autónoma de Guerrero.

Análisis de datos. La riqueza se consideró como el número de especies de moluscos presentes. La diversidad de moluscos se midió con el índice de Shannon-Wiener. La densidad se evaluó en organismos $/ \mathrm{m}^{2}$. La frecuencia de aparición se determinó como el número de recolectas, expresándola en porcentaje donde se presentó una especie. El análisis de dominancia simple se basó en el total de organismos de cada especie con respecto al total de organismos de todas las especies, expresada en porcentaje. La determinación del conjunto de las especies con mayor importancia relativa acumulada se realizó tomando como criterio aquellas que en conjunto sumaran la mejor aproximación al $90 \%$ de la dominancia total.

La precipitación pluvial total $(\mathrm{mm})$, así como la diversidad de moluscos (H'), se relacionaron con la densidad de una especie de gasterópodo y una de bivalvo, consideradas como las especies más importantes y representativas de la comunidad; esta relación se midió por medio de la prueba de correlación de Pearson, a un nivel de confianza de $95 \%$, observando las tendencias con 
un gráfico de dispersión de puntos.

\section{Resultados}

En Playa Troncones se encontraron 42 especies de moluscos, pertenecientes a 34 géneros y 23 familias. La riqueza de especies se distribuyó de la siguiente manera: en diciembre de 2000 se registraron 24 especies; en marzo de 2001 se registraron 27, en junio 24, en septiembre 20 y en diciembre 26 especies. Se identificaron 33 especies de gasterópodos, 8 de bivalvos y una de poliplacóforos. Se registraron 12 especies con $100 \%$ de frecuencia de aparición en las muestras y 8 con el $80 \%$ (Cuadro 1).

La densidad promedio durante el estudio fue de 74.02 organismos $/ \mathrm{m}^{2}$. Los valores de la densidad promedio de organismos y de la precipitación pluvial total durante las recolectas (Fig. 1) se relacionaron de manera gráfica. La tendencia observada fue que al aumentar la precipitación total se registró una disminución en la densidad promedio de los organismos, siendo en la recolecta de septiembre de 2001, la que correspondió a la época de ciclones y huracanes, cuando más se marcó dicha tendencia. Sin embargo, el periodo en que se realizó el estudio se caracterizó porque su precipitación estuvo por debajo del promedio que se ha registrado para la región, por lo que se puede considerar un año seco.

Brachidontes semilaevis, Nerita funiculata, Isognomon janus, Plicopurpura pansa, Nerita scabricosta, Petaloconchus complicatus y Nodilittorina aspera, se registraron como el conjunto de especies de mayor importancia relativa acumulada en el ciclo anual en que se llevó a cabo el estudio.

El conjunto de las especies con mayor importancia

Cuadro 1. Especies de moluscos y frecuencia de aparición (FA) en el mesolitoral rocoso de Playa Troncones, Guerrero, México; diciembre 2000-diciembre 2001

FA\% FA\%

\begin{tabular}{|c|c|c|c|}
\hline \multicolumn{3}{|c|}{$F A \%$} & \multirow[t]{2}{*}{$F A \%$} \\
\hline \multicolumn{3}{|c|}{ GASTROPODA } & \\
\hline Lottia mitella (Menke, 1847) & 20 & Mancinella speciosa (Valenciennes, 1832) & 80 \\
\hline L. mesoleuca (Menke, 1851) & 100 & M. triangularis (Blainville, 1832) & 80 \\
\hline Tectura filosa (Carpenter, 1865) & 20 & Stramonita haemastoma (Linnaeus, 1758) & 80 \\
\hline Fissurella asperella Sowerby, 1835 & 20 & Thais kiosquiformis (Duclos, 1832) & 100 \\
\hline F. oscura Sowerby, 1835 & 20 & T. melones (Duclos, 1832) & 20 \\
\hline Astraea buschii (Philippi, 1844) & 20 & Plicopurpura columellaris (Lamark, 1822) & 20 \\
\hline Tegula maculostriata (C.B. Adams, 1845) & 20 & P. pansa (Gould, 1853) & 100 \\
\hline Nerita scabricosta Lamark, 1822 & 100 & Cantharus sanguinolentus (Duclos, 1846) & 40 \\
\hline N. funiculata Menke, 1851 & 80 & Leucozonia cerata (Wood, 1828) & 40 \\
\hline Nodilittorina aspera (Philippi, 1846) & 100 & Opeatostoma pseudodon (Burrow, 1815) & 100 \\
\hline N. modesta (Philippi, 1846) & 80 & Columbella fuscata Sowerby, 1832 & 60 \\
\hline Petaloconchus complicatus Dall 1908 & 100 & Mitrella ocellata (Gmelin, 1791) & 100 \\
\hline P. macrophagma Carpenter, 1857 & 80 & Mitra tristis Broderip, 1836 & 40 \\
\hline Cerithium menkei Carpenter, 1857 & 40 & Conus nuх Broderip, 1833 & 40 \\
\hline Liocerithium judithae Keen, 1971 & 20 & Hoffmannola hansi Marcus \& Marcus, 1967 & 100 \\
\hline Hipponix a. panamensis C.B. Adams, 1852 & 20 & Siphonaria gigas Sowerby, 1825 & 20 \\
\hline Pilosabia pilosa (Deshayes, 1832) & 20 & S. palmata Carpenter, 1857 & 100 \\
\hline \multicolumn{4}{|c|}{ BIVALVIA } \\
\hline Arca mutabilis (Sowerby, 1833) & 20 & Saccostrea palmula (Carpenter, 1857) & 20 \\
\hline Brachidontes semilaevis (Menke, 1849) & 100 & Carditamera affinis Sowerby, 1833 & 20 \\
\hline Isognomon janus Carpenter, 1857 & 100 & Chama echinata Broderip, 1835 & 80 \\
\hline Undulostrea megodon (Hanley, 1846) & 20 & & \\
\hline \multicolumn{4}{|c|}{ POLYPLACOPHORA } \\
\hline Chiton articulatus Sowerby, 1832 & 80 & & \\
\hline
\end{tabular}




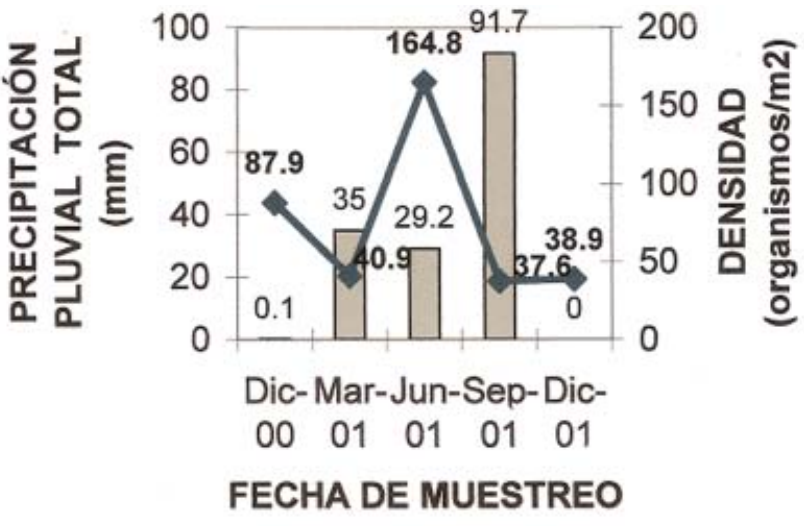

\section{JPRECIPITACIÓN •DENSIDAD}

Figura 1. Valores de la densidad promedio de organismos y de la precipitación total observada durante los muestreos.

relativa acumulada en cada recolecta fluctuó de 4 a 9 , entre junio y septiembre de 2001. Sólo B. semilaevis y $P$. pansa fueron parte del conjunto de las especies con mayor importancia relativa acumulada y que además presentaron el $100 \%$ de frecuencia de aparición (Cuadro 2).

La relación entre la densidad del caracol de tinte $P$. pansa y la precipitación total registró una alta correlación positiva $(0.733, \mathrm{P}>0.05)$, lo que indica una fuerte tendencia a aumentar la densidad de dichos caracoles en las fechas cuando se produjeron lluvias copiosas (Fig. 2). Respecto a la relación entre la precipitación y la densidad del bivalvo $B$. semilaevis, se observó una fuerte correlación negativa
(-0.596, $\mathrm{P}>0.05)$, lo cual indica que, al contrario de la especie carnívora $P$. pansa, las densidades de $B$. semilaevis disminuyen conforme va aumentando el nivel o la cantidad de precipitación pluvial (Fig. 3).

El índice de diversidad $\left(\mathrm{H}^{\prime}\right)$ para todo el estudio fue 2.82 bits/ind, aunque fluctuó de 1.97 bits/ind. en junio a 3.21 bits/ind., en diciembre de 2001. La relación gráfica entre la diversidad y la precipitación total registrada por recolecta (Fig. 4) presentó un proceso inverso al que se observó entre densidad y precipitación total, dado que al aumentar el volumen de precipitación, también aumentó el índice de diversidad biológica; excluyendo de estas observaciones los datos registrados en diciembre 2001, cuando una disminución drástica de la precipitación no se reflejó en una disminución de la diversidad.

Por lo que respecta a los valores de diversidad encontrados (H') y la densidad del caracol P. pansa, se registró una alta correlación positiva $(0.708, \mathrm{P}>0.05)$, lo cual muestra que a medida que la diversidad va en aumento se incrementa el número de ejemplares del caracol de tinte (Fig. 5). Por otro lado, la correlación entre la diversidad y la densidad del bivalvo B. semilaevis, registró un alto valor negativo estadísticamente significativo $(-0.880, \mathrm{P}<0.05)$; esto es, a medida que el valor de la diversidad aumenta, la densidad del bivalvo se reduce significativamente (Fig. 6).

\section{Discusión}

La riqueza de moluscos encontrada en un ciclo anual en Playa Troncones, es similar a lo registrado por Flores et

Cuadro 2. Especies dominantes por fecha de recolecta y su dominancia simple (\%) en el mesolitoral rocoso de Playa Troncones, Guerrero, México

\begin{tabular}{lrrrrr}
\hline Especie & Dic-00 & Mar-01 & Jun-01 & Sep-01 & Dic-01 \\
\hline Brachidontes semilaevis & 49.71 & 33.13 & 54.67 & 10.50 & 23.65 \\
Isognomon janus & 29.01 & 16.26 & & & 23.13 \\
Plicopurpura pansa & 7.34 & 11.24 & 6.34 & 26.72 & 16.19 \\
Nerita scabricosta & 3.47 & & 3.65 & 28.32 & 2.82 \\
Petaloconchus complicatus & 2.90 & 10.88 & & 5.45 & 11.31 \\
Nodilittorina aspera & & 9.65 & & 8.64 & \\
Petaloconchus macrophagma & & 7.33 & & & 5.27 \\
Siphonaria palmata & 5.01 & & & \\
Mitrella ocellata & & & & 3.92 & 2.18 \\
Hoffmannola hansi & & & & 3.59 \\
Chiton articulatus & & & & & 2.18 \\
Mancinella triangularis & & & 26.74 & 3.85 & \\
Nerita funiculata & & & 91.38 & 91.62 & 90.36 \\
$\quad$ Dominancia acumulada & 92.43 & 93.52 & & & \\
\hline
\end{tabular}




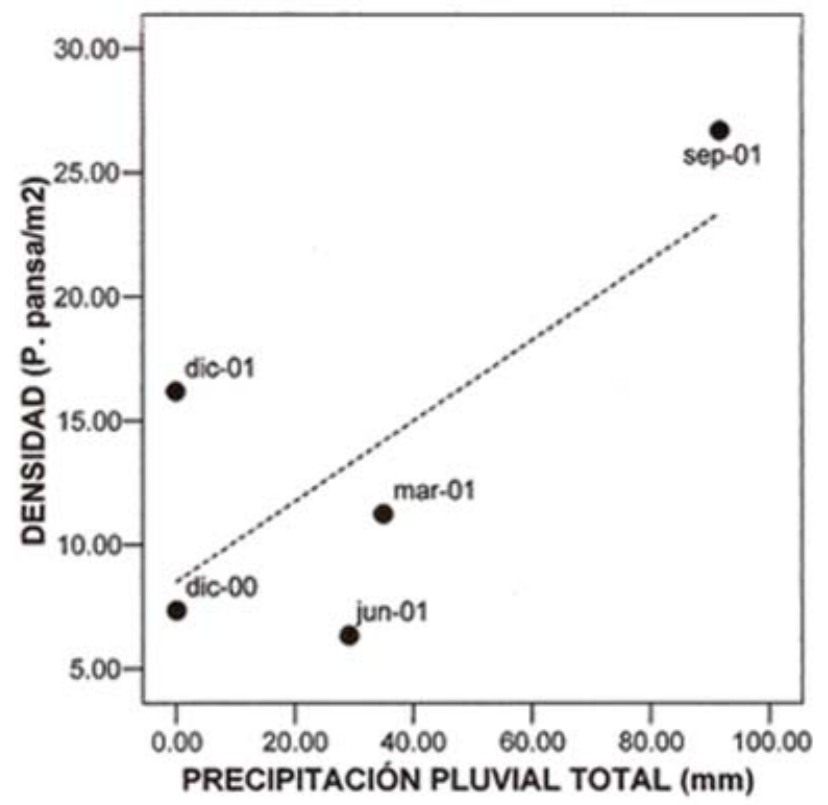

Figura 2. Dispersión y línea de tendencia de la relación entre la densidad del caracol Plicopurpura pansa y la precipitación total.

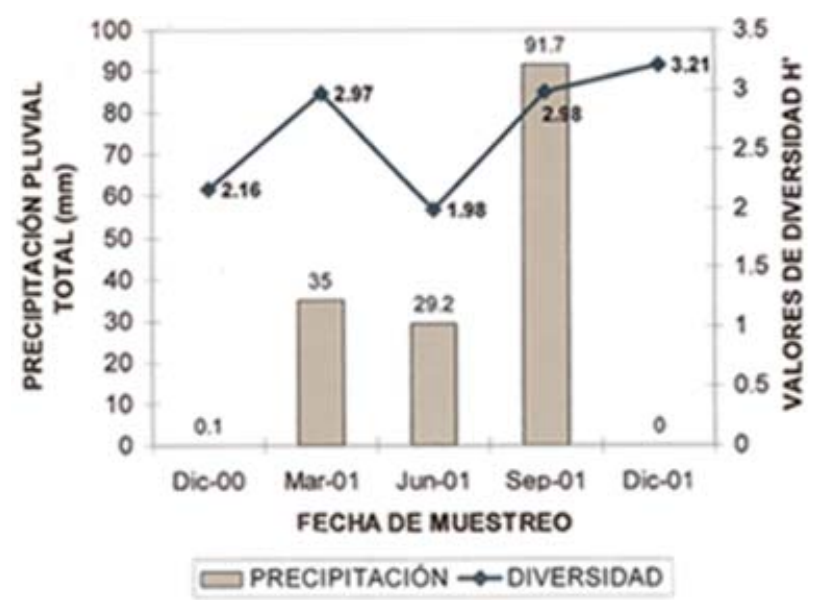

Figura 4. Relación entre la diversidad y la precipitación total registrada por fecha de muestreo.

al. (2003), para la isla La Roqueta en Acapulco, Guerrero, donde encontraron 44 especies en el mesolitoral rocoso de 2 playas con diferente exposición al oleaje. En cuanto a riqueza de moluscos, también existe semejanza con los datos de Esqueda et al. (2000) para Bahía Cuastecomate, Jalisco, que registraron 44 especies en 5 playas rocosas, cuyas características de exposición al oleaje fueron diferentes y sus muestreos se realizaron en las zonas

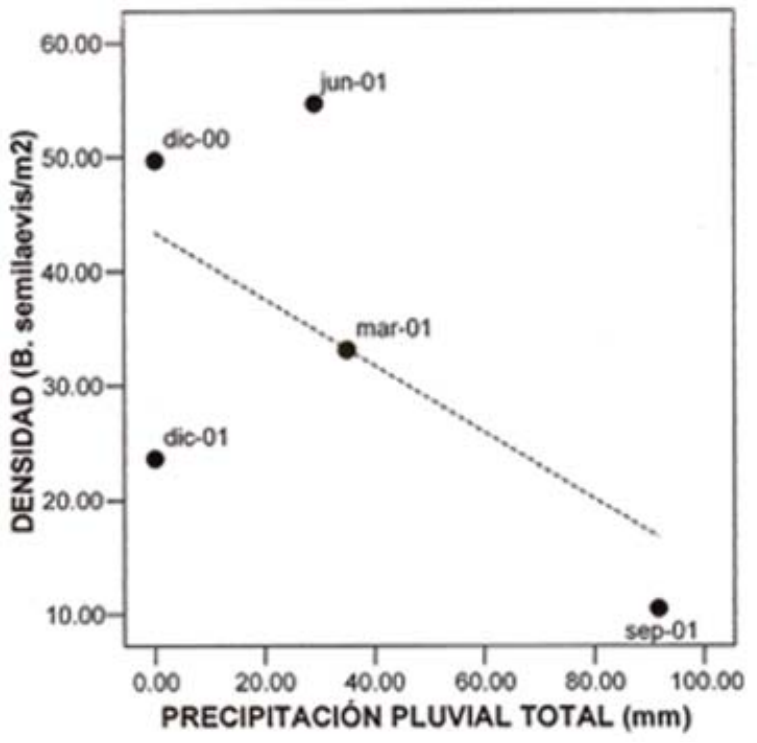

Figura 3. Dispersión y línea de tendencia de la relación entre la densidad del bivalvo Brachidontes semilaevis y la precipitación total.

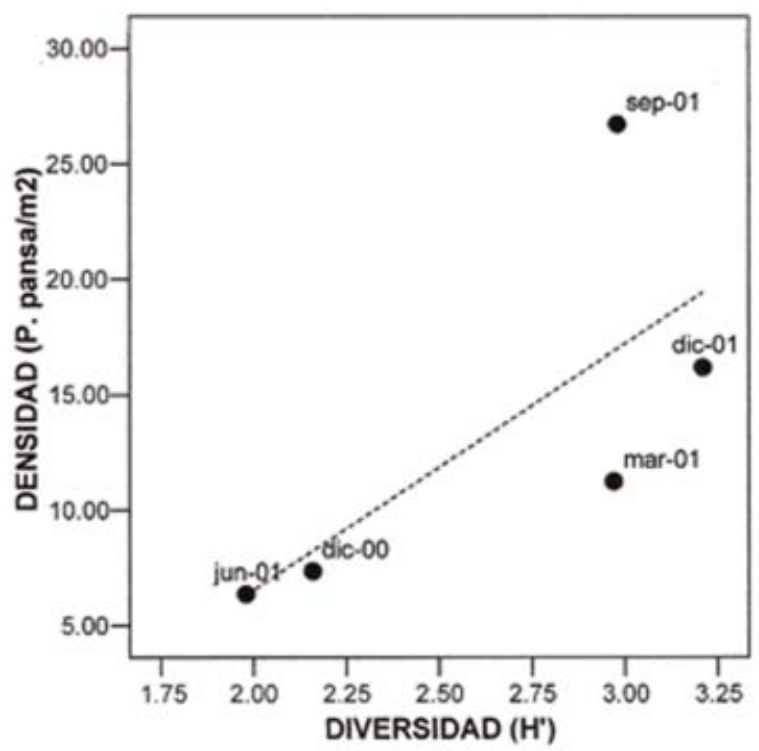

Figura 5. Dispersión y línea de tendencia de los valores de diversidad registrados (H') y la densidad del caracol Plicopurpura pansa.

mesolitoral y supralitoral. Otros estudios sobre diversidad malacológica realizados en el estado de Guerrero, como los de Villalpando (1986), Salcedo et al. (1988) y Delgado (1989) encontraron un mayor número de especies de moluscos; sin embargo, la amplitud de la zona en 


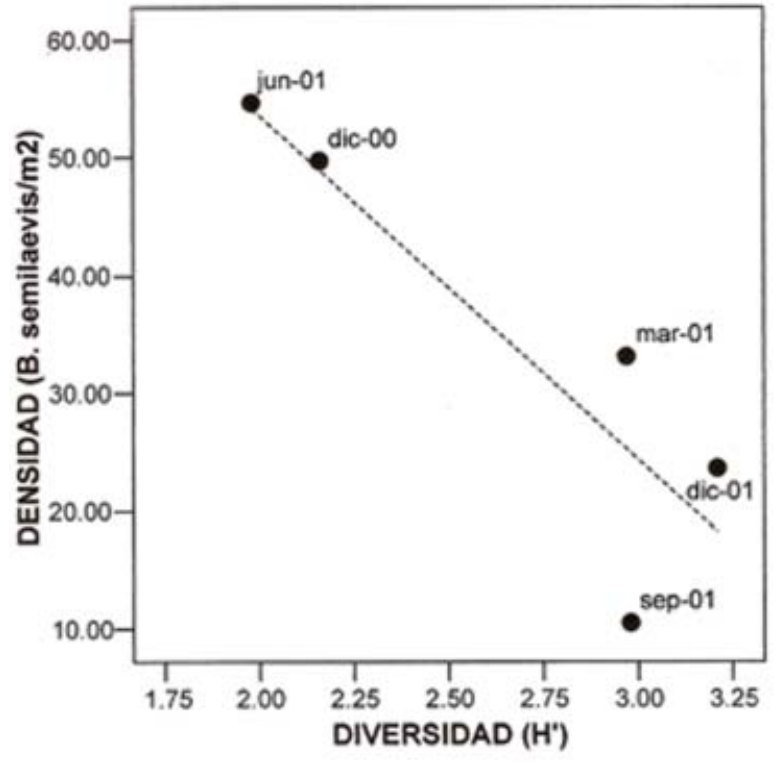

Figura 6. Dispersión y línea de tendencia de los valores de diversidad registrados (H') y la densidad del bivalvo Brachidontes semilaevis.

que tomaron la muestra abarcó del supralitoral hasta el infralitoral.

La clase Gastropoda presentó la mayor riqueza, seguida por Bivalvia y por último Polyplacophora, lo que concuerda con Castillo-Rodríguez y Amezcua-Linares (1992), Del Río y Villarroel (2000) y Flores et al. (2003).

Dado que B. semilaevis, $N$. funiculata, I. janus, $P$. pansa, $N$. scabricosta, $P$. complicatus y $N$. aspera se presentaron como el conjunto de especies de mayor importancia relativa acumulada en el ciclo anual, se les considera como representativas de la comunidad malacológica del mesolitoral rocoso de Playa Troncones. De este conjunto de especies, $B$. semilaevis y $P$. pansa presentaron el mayor porcentaje en la frecuencia de aparición, por lo que se concluye que son las de mayor importancia en esta comunidad. Sin embargo, $P$. pansa es un organismo móvil $\mathrm{y}$ una especie de mayor tamaño que $B$. semilaevis que es sésil y forma agregaciones generalmente muy densas.

Las especies B. semilaevis, $N$. aspera, I. janus, $P$. pansa, $M$. triangularis se encontraron entre las más densas del estudio; también han sido registradas con altas densidades por Román et al. (1991), Castillo-Rodríguez y Amezcua-Linares (1992), Esqueda et al. (2000) y Del Río y Villarroel (2001).

Los valores de diversidad encontrados en el mesolitoral rocoso Playa Troncones indican una alta diversidad malacológica, comparable con los de Acapulco (Delgado, 1989) y la isla La Roqueta, en dos localidades protegidas
(García, 1994). Los valores en Playa Troncones fueron más altos que lo registrado en diferentes épocas en Jalisco (Román et al., 1991); con estos datos podemos concluir que la malacofauna en la zona de estudio es altamente diversa y similar a los valores de diversidad que se han encontrado para regiones tropicales.

En la zona de estudio se observó la tendencia en el aumento de la precipitación pluvial total con disminución en la densidad promedio de los organismos, marcándose con mayor fuerza este comportamiento en la época de ciclones y huracanes.

Se registró una alta correlación positiva entre la densidad del carnívoro $P$. pansa y la precipitación total, lo que reveló que en las fechas de lluvias copiosas, la densidad de estos caracoles se incrementó. En contraste, el bivalvo $B$. semilaevis presentó correlación negativa, al observarse que las densidades disminuyeron conforme aumenta la precipitación.

La relación entre diversidad y precipitación total, presentó un proceso inverso a la relación entre densidad y precipitación total, ya que al aumentar la precipitación, aumentó la diversidad biológica.

La relación entre los valores de diversidad y la densidad de la población de $P$. pansa registrada en este estudio mostró que la densidad de este molusco aumentó conforme los valores de diversidad aumentaron. Sin embargo, $B$. semilaevis reveló un comportamiento contrario al caracol carnívoro; esta correlación negativa de la densidad poblacional del bivalvo con respecto a la diversidad indica el aumento de la posible competencia por el sustrato al aumentar la precipitación y con ésta la diversidad, lo que posiblemente impacte en la disminución de la densidad del bivalvo. Aunque sería difícil llegar a una conclusión en este sentido, también se puede interpretar que al aumentar la precipitación pluvial y disminuir la densidad total deberá de haber mayor espacio y menor competencia. Quizás la causa de disminución del bivalvo durante las lluvias no sea la competencia, sino el estrés por la disminución de la salinidad que causan las lluvias junto con los periodos de desecación y mayor temperatura durante el verano.

\section{Literatura citada}

Arriaga, C. L., E. Vázquez D., J. González C., R. Jiménez R., E. Muñoz L. y B. Aguilar S. 1998. Regiones prioritarias marinas de México. Comisión Nacional para el Conocimiento y Uso de la Biodiversidad, México, D.F. 198 p.

Baqueiro, E. 1979. Sobre la distribución de Megapitaria aurantiaca (Sowerby), M. squalida (Sowerby) y Dosinia ponderosa (Gray) en relación a la 
granulometría del sedimento (Bivalvia: Veneridae): Nota científica. Anales del Centro de Ciencias del Mar y Limnología, Universidad Nacional Autónoma de México 6:25-32.

Baqueiro, E. y J. Stuardo. 1977. Observaciones sobre la biología, ecología y explotación de Megapitaria aurantiaca (Sowerby, 1831), M. squalida (Sowerby, 1835) y Dosinia ponderosa (Gray, 1838) (Bivalvia: Veneridae) de la bahía de Zihuatanejo e isla Ixtapa, Guerrero, México. Anales del Centro de Ciencias del Mar y Limnología, Universidad Nacional Autónoma de México 4:161-208.

Briggs, J. C. 1974. Marine zoogeography. Mc Graw-Hill, New York. 475 p.

Brower, J. E., J. H. Zar y C. N. Von Ende. 1998. Field and laboratory methods for general ecology, cuarta edition, WCB/McGraw-Hill, New York. 271 p.

Carranza-Edwards, A., M. Gutiérrez-Estraday R. RodríguezTorres. 1975. Unidades morfo-tectónicas continentales de las costas mexicanas. Anales del Instituto de Ciencias del Mar y Limnología, Universidad. Nacional Autónoma de México 2:81-88.

Castillo-Rodríguez, Z. G. y F. Amezcua-Linares. 1992. Biología y aprovechamiento del caracol morado Plicopurpura pansa (Gould 1853) (Gastropoda: Neogastropoda) en la costa de Oaxaca, México. Anales del Instituto de Ciencias del Mar y Limnología, Universidad Nacional Autónoma de México 19:223-234.

Delgado-Blas, V. H. 1989. Estudio sistemático y aspectos ecológicos de gasterópodos de la facie rocosa de la Bahía de Acapulco, Guerrero, México. Tesis, Escuela Superior de Ecología Marina, Universidad Autónoma de Guerrero, Acapulco. 97 p.

Del Río, Z. O. y M. M. Villarroel. 2001. Variación estacional de moluscos en las pozas de marea del faro de Brucerías, Michoacán, México. Resúmenes, VIII Reunión Nacional de Malacología y Conquiliología, Ciudad Victoria, Tamps. México. Facultad de Biología, Universidad Michoacana de San Nicolás de Hidalgo, Morelia, Michoacán. p. 26-28.

Esqueda, M. C., E. Ríos-Jara, J.E. Michel-Morfín y V. Landa-Jaime. 2000. The vertical distribution and abundance of gastropods and bivalves from rocky beaches of Cuastecomate Bay, Jalisco. Revista de Biología Tropical 48:765-775.

Flores-Rodríguez, P., R. Flores-Garza, S. García-Ibáñez y A Valdés-González. 2003. Riqueza y diversidad de la malacofauna del mesolitoral rocoso de la Isla la Roqueta, Acapulco, Guerrero, México. Ciencia, Revista de Investigación Científica, Universidad Autónoma de Guerrero 11:5-14.
García, E. 1981. Modificaciones al sistema de clasificación climática de Köpen (para adaptarlo a las condiciones de la República Mexicana), Instituto de Geografía, Universidad Nacional Autónoma de México, México, D.F. 246 p.

García-López, J. A. 1994. Fauna malacológica de acompañamiento del caracol Purpura pansa Gould 1853 en la zona mesolitoral de la isla Roqueta, Acapulco, Guerrero, México. Tesis, Escuela Superior de Ecología Marina, Universidad Autónoma de Guerrero, Acapulco. 97 p.

Holguín-Quiñones, O. E. y A. C. González-Pedraza. 1989. Moluscos de la franja costera del estado de Oaxaca, México. Atlas 7, Centro Interdisciplinario de Ciencias Marinas, Dirección de Bibliotecas y Publicaciones, Instituto Politécnico Nacional, México, D.F. 221 p.

Holguín-Quiñones, O. E. y A. C. González-Pedraza. 1994. Moluscos de la franja costera de Michoacán, Colima y Jalisco, México. Dirección de Bibliotecas y Publicaciones, Instituto Politécnico Nacional, México, D.F. 133 p.

Keen, A. M. 1971. Sea shells of tropical West America, segunda edición, Stanford University Press, California. $1064 \mathrm{p}$.

Landa-Jaime, V. y J. Arciniega-Flores. 1998. Macromoluscos bentónicos de fondos blandos de la plataforma continental de Jalisco y Colima, México. Ciencias Marinas 24:155-167.

Mottana, A., R. Crespi y G. Liborio. 1980. Guía de minerales y rocas, segunda edición, Grijalbo, Barcelona, 605 p.

Olabarría, C. 1999. Estructura y variación estacional de poblaciones de moluscos asociadas a la pesca artesanal de langosta en el Pacífico tropical. Revista de Biología Tropical 47:851-865.

Pérez-Peña, M. y E. Ríos-Jara E. 1998. Moluscos gastrópodos de la plataforma continental de Jalisco y Colima, México: especies recolectadas con red de arrastre. Ciencias Marinas 24:425-442.

Reguero, M. y A. García-Cubas. 1989. Moluscos de la plataforma continental de Nayarit: sistemática y ecología (cuatro campañas oceanográficas). Anales del Centro de Ciencias del Mar y Limnología, Universidad Nacional Autónoma de México 16:33-58.

Ríos-Jara, E., Pérez-Peña M., Beas-Luna R., López-Uriarte E. y E. Juárez-Carrillo. 2001. Gastropods and bivalves of commercial interest from the continental shelf of Jalisco and Colima, México. Revista de Biología Tropical 49:785-789.

Román C. R., F. M. Cruz A. y A. L. Ibáñez A. 1991. Observaciones ecológicas de los moluscos de la zona intermareal de la bahía de Chamela, Jalisco, México. Anales del Instituto de Biología, Universidad Nacional. 
Autónoma de México, Serie Zoología 62:17-32.

Salcedo-Martínez, S., G. Green, A. Gamboa y P. Gómez. 1988. Inventario de macroalgas y macroinvertebrados bénticos, presentes en áreas rocosas de la región de Zihuatanejo, Guerrero, México. Anales del Instituto Ciencias del Mar y Limnología, Universidad Nacional Autónoma de México 15:73-96.

Scheaffer, R. L., W. Mendenhall y L. Ott. 1987. Elementos de muestreo, tercera edición, Iberoamericano, México, D.F. 321 p.

Sevilla, M. L. 1995. Moluscos de la franja costera de Chiapas, México. Escuela Nacional de Ciencias Biológicas, Instituto Politécnico Nacional, México, D.F. 152 p.

Skoglund, K. 1991. Additions to the Panamic Province Bivalve (Mollusca) literature 1971 to 1990 . The Festivus 22 (Suppl. 2):63.

Skoglund, K. 1992. Additions to the Panamic Province
Gasteropods (Mollusca) literature 1971 to 1992. The Festivus 24 (Suppl.):169.

Stephenson, T. A. y A. Stephenson. 1949. The universal features of zonation between tidemarks and rocky coasts. Journal of Ecology 37:289-305.

Stuardo, J. y M. Villarroel. 1976. Aspectos ecológicos y distribución de los moluscos en las lagunas costeras de Guerrero, México. Anales del Centro de Ciencias del Mar y Limnología, Universidad Nacional Autónoma de México 3:65-92.

Villalpando-Canchola, E. 1986. Diversidad y zonación de moluscos de superficie rocosa, Isla Roqueta, Acapulco, Guerrero. Tesis, Facultad de Ciencias, Universidad Nacional Autónoma de México, México, D.F. 150 p.

Villarroel, M. M., A. Magaña M., B. Gómez C., O. Del Río Z., J. Lucio P. y J. Sánchez S. 2000. Diversidad de moluscos en el litoral rocoso de Michoacán, México. Mexicoa 2:54-63. 